\title{
Turbulent Structure of Supersonic Flowfield with Transverse Injection*
}

\author{
Shohei URAMOTO**, Toshinori KOUCHI** and Goro MASUYA** \\ **Department of Aerospace Engineering, Tohoku University \\ 6-6-01 Aramaki-aza-Aoba, Aoba-ku, Sendai, Miyagi 980-8579, Japan \\ E-mail: uramoto@scrj.mech.tohoku.ac.jp
}

\begin{abstract}
We investigated a supersonic flowfield with transverse injection of air or helium by stereoscopic particle image velocimetry and calculated single-time two-point spatial correlations of velocity fluctuations. For both injection gases, the bow shock wave formed by the injection and its reflection from the upper wall could be seen in the mean velocity distribution. In the velocity fluctuation intensity distribution, we observed vibrating counter-rotating vortex pair and the Reynolds stress was formed along the track of the maximum mean velocity gradient. Correlations showed the scale structure of turbulence and the shapes of the correlation region changed with the selected velocity component. Differences were observed between the results obtained for the two injectant species.
\end{abstract}

Key words: Spatial Correlation, Velocity Fluctuation, Jet in Supersonic Crossflow, Stereoscopic Particle Image Velocimetry

\section{Introduction}

The supersonic combustion ramjet (scramjet) engine has been studied and developed as a propulsion system for hypersonic flight vehicles. Flow velocity in a combustor of this engine is supersonic and the time for mixing between air and fuel is very short. Therefore, fuel injection and mixing are required to be highly efficient.

Transverse injection into a supersonic flow is a typical fuel injection method. This injection method causes a large loss, but it results in high penetration of the jet into the mainflow and introduces three-dimensional disturbances to accelerate mixing.

Gruber et al. [1] examined the transverse injection structure by using Mie scattering, and Takahashi et al. [2] measured the flowfield by using the acetone planer laser-induced fluorescence (PLIF) technique. The single-time two-point spatial correlation of instantaneous fluorescence intensity is proportional to the acetone molar concentration, and calculations of these spatial correlations along the $50 \%$ averaged track of the mean concentration distribution can reveal the turbulent structure of the scalar field [2]. Takahashi et al. [3] also investigated the effect of injection species on the turbulent structures using the same acetone PLIF technique. They found that the shapes of turbulent structures, such as angle and length, changed with gas species.

In the above studies, the scalar field and the scalar structure of jet were investigated. The scalar structures were driven by the velocity field and the velocity fluctuation was the main source of turbulent mixing. Therefore, to investigate the jet structure and understand turbulent mixing in more detail, it is highly desirable to measure instantaneous velocity fluctuations and investigate their characteristic features.

In this study, we investigated the mean and instantaneous velocity field of a supersonic 
flow with transverse sonic injection by stereoscopic particle image velocimetry (SPIV) and calculated single-time two-point spatial correlations of the velocity fluctuations to understand the flow structure.

\section{Experimental Setting and Conditions}

\section{Test facility}

Figure 1 shows a schematic diagram of the experimental apparatus used in this investigation. We used a suction-type supersonic wind tunnel. Unheated atmospheric air was inhaled into a vacuum tank through a Mach 2 two-dimensional Laval nozzle and test

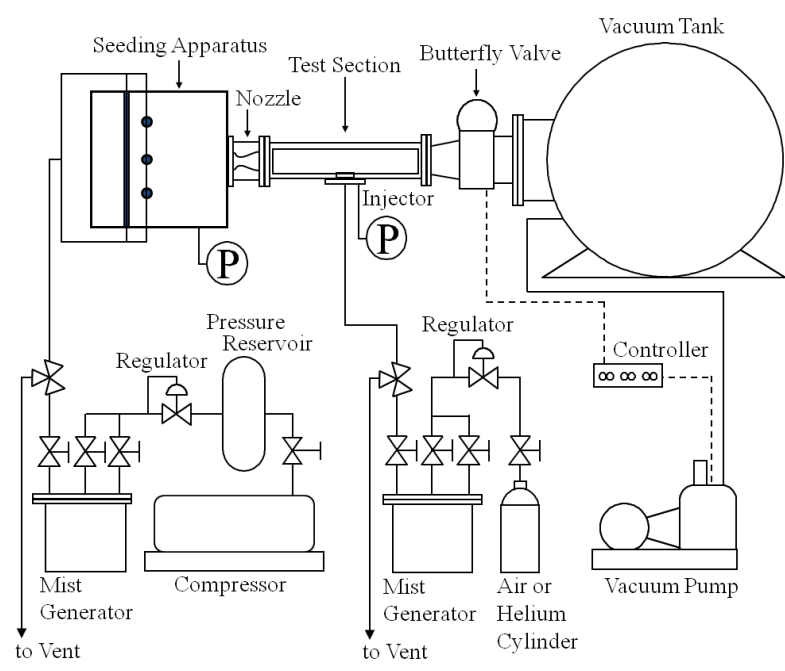

Fig. 1 Experimental apparatus section. The tank had a volume of $8 \mathrm{~m}^{3}$. A seeding chamber was placed at the entrance of the nozzle to supply tracer particles into the mainstream for the SPIV.

The test section was 290 $\mathrm{mm}$ long and had a square cross section (side length: 30 $\mathrm{mm})$. The walls of the test section were made of transparent acrylic resin. Red acrylic resin was used for the lower wall with the injection hole to reduce the influence of reflection of the laser light

sheet. The diameter of the injection hole was $2.5 \mathrm{~mm}$. It was located on the center line of the lower wall at a point $75 \mathrm{~mm}$ downstream from the entrance. A schematic diagram of the test section and the injection orifice is shown in Fig. 2.

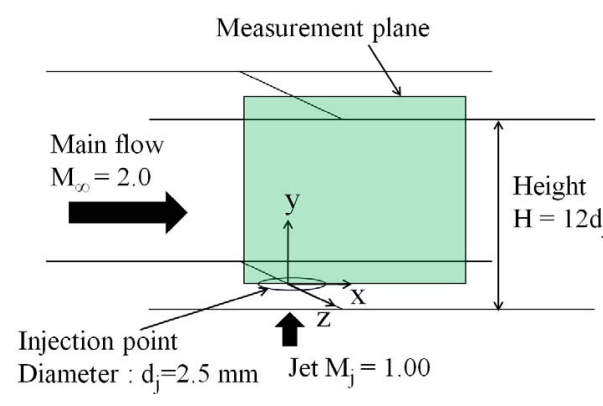

Fig. 2 Schematic of the measurement region and coordinates
The injectant gases were pressurized air or helium fed from a cylinder. The pressurized gas passed through a Laskin nozzle [4-5] in a mist generator which produced the tracer particles. We used two mist generators: one fed tracer particles to the seeding chamber of the main flow and the other supplied them to the injector. The tracer particles were droplets of dioctyl sebacate (density: $913.5 \mathrm{~kg} / \mathrm{m}^{3}$ ). The diameter of the tracer particle was measured to be about $1 \mu \mathrm{m}$ by Kitamura [6] and confirmed by Koike et al. [7]. The jet-to-mainstream momentum flux ratio $J$ was $1.96 \pm 0.02$ for air and $1.99 \pm 0.02$ for helium. These values were selected by referring to Takahashi et al. [2].

\section{Stereoscopic PIV}

We used the double-frame, double-exposure mode of the SPIV system to observe the particle side scattering. A schematic arrangement of the CCD camera setup is shown in Fig. 3. A double-pulse Nd:YAG laser (532 nm, $15 \mathrm{~mJ} /$ pulse, $15 \mathrm{~Hz}, 5-7 \mathrm{~ns}$ pulse duration) was used as a light source. 


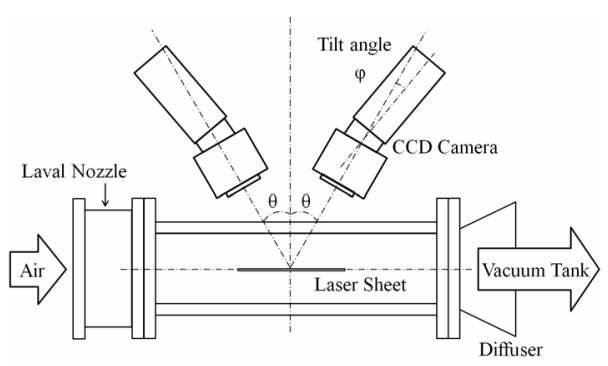

Fig. 3 CCD camera setup (Scheinpflug setup)
Two cylindrical lenses and a mirror were used to produce a laser sheet that was $50 \mathrm{~mm}$ wide and a little less than $1 \mathrm{~mm}$ thick. The separation time between two laser pulses was 500 ns. In this measurement plane, the mean spanwise velocity was almost zero and the root mean square of spanwise velocity was about $100 \mathrm{~m} / \mathrm{s}$. The laser sheet was more than four times thicker than the spanwise length of tracer particle tracks [8]. Therefore, this laser sheet had an appropriate thickness.

Light scattered from the particles was recorded with the two CCD cameras (1600 pixels $\times 1200$ pixels). The laser and cameras were synchronized with a pulse generator. Data of 8-bit monochrome images were transferred to a PC and stored. The image data were processed to calculate the velocity vectors using the direct cross-correlation method in an analysis program (FtrPIV). We eliminated the error vectors by using the median filter. The transformation coefficient between image and real length was $30.019 \mu \mathrm{m} / \mathrm{pixel}$. The other measurement conditions for SPIV are listed in Table 1.

Table 1 Measurement conditions for SPIV

\begin{tabular}{ccccc}
\hline \hline & Camera angle & $\theta$ & 27 & {$[\mathrm{deg}]$} \\
Imaging system & Tilt angle & $\varphi$ & 8 & {$[\mathrm{deg}]$} \\
& Aperture & 6.7 & {$[-]$} \\
& Number of images & 1500 & {$[\mathrm{pair}]$} \\
\hline \multirow{4}{*}{ Correlation system } & Interrogation area & $33 \times 33$ & {$\left[\mathrm{pixel}^{2}\right]$} \\
& Overlap & 51 & {$[\%]$} \\
& Search region & \pm 15 & {$[\mathrm{pixel}]$} \\
& Correlation threshold & 0.2 & {$[-]$} \\
& Luminance threshold & $10 / 256$ & {$[-]$} \\
\hline \multirow{3}{*}{ Calibration target } & Anchoring points & $40 \times 25$ & {$[-]$} \\
& Anchoring point interval & 1.18 & {$[\mathrm{~mm}]$} \\
& Anchoring point diameter & 0.5 & {$[\mathrm{~mm}]$} \\
\hline \hline
\end{tabular}

In order to check the traceability of the particle, we calculated Kolmogorov time and length scales. We assumed that this flow is isotopic turbulent flow, thus we used following equations [9].

$$
\frac{\eta}{l}=\left(\frac{1}{A}\right)^{1 / 4}\left(\frac{\sqrt{\overline{u^{\prime 2}}} l}{v}\right)^{-3 / 4} \text { and } \tau=\frac{\eta^{2}}{v}
$$

where $l$ is the integral scale which is estimated from the spatial correlation, $\eta$ is the Kolmogorov length scale, $\tau$ is the Kolmogorov time scale, $A$ is a constant of $O(1)$, $\sqrt{u^{\prime 2}}$ is measured velocity fluctuation intensity and $v$ is dynamic viscosity which is calculated assuming isentropic relation for the main stream. We used the following values: $l$ was about $1 \mathrm{~mm}, A$ was $1, \sqrt{u^{\prime 2}}$ was about $20 \mathrm{~m} / \mathrm{s}$ and $v$ was about $4.0 \times 10^{-5} \mathrm{~m}^{2} / \mathrm{s}$. Then, we obtained that the Kolmogorov length scale was about $10 \mu \mathrm{m}$ and the Kolmogorov time scale was about $2.5 \mu \mathrm{s}$. On the other hand, the time constant of the particle delay was estimated from the experimental data of the particle motion through the normal shock wave in Mach 1.5 flow [7]. The result was about $4 \mu \mathrm{s}$. The Kolmogorov time scale and the time constant of particle delay were similar order. However, the interrogation area that is about $1 \mathrm{~mm} \times 1$ $\mathrm{mm}$ is much bigger than the Kolmogorov length scale. Therefore, we could not capture a motion of Kolmogorov length scale. 
Next, for calculation of this measurement uncertainty, we conducted the uncertainty analysis. We consulted the method of the reference book [8] to calculate the uncertainties of our SPIV measurements. In this uncertainty analysis, there are some uncertainty components such as calibration accuracy, orthogonality of laser sheet, laser power distribution, smoothing in interrogation area, error vector, subpixel error, trigger pulse and laser timing. In these components, orthogonality of laser sheet and subpixel error had big influence on the uncertainty. In this experiment, uncertainty was about $2.5 \%$ of the mainstream velocity for the streamwise and heightwise directions and was about $5 \%$ for the spanwise direction.

Finally, a validation analysis of this SPIV system has been performed [10]. In this validation, we obtained the streamwise and heightwise velocity fluctuation intensities $\overline{u^{\prime 2}}$ and $\overline{v^{\prime 2}}$, respectively, and the Reynolds stress $\overline{u^{\prime} v^{\prime}}$ of the boundary layer. We compared our results with data from other measurement techniques and DNS calculation. Our data agreed quantitatively with those of other researchers.

As a result, we could not capture a motion of the Kolmogorov length scale, but we could measure the large scale motion and could obtain the velocity fluctuation intensities and the Reynolds stress.

\section{Results and Discussion}

\section{Mean velocity distribution}

Figures 3 and 4 show the mean velocity distributions of each component. All velocities were nondimensionalized by using the mainstream velocity, $\bar{u}$ main. Some parts in the figures are blank because, in these regions, the amount of data excluding erroneous measurements was insufficient to calculate mean or turbulent characteristics or because no clear images were obtained due to grime on the test section walls from the dioctyl sebacate.

In Figs. 3 and 4, we can see the bow shock wave formed by the injection and its reflection from the upper wall.

For the helium injection case shown in Fig. 4 (b), the mean heightwise velocity component $\bar{v}$ had a maximum value around the point $\left(x / d_{j}, y / d_{j}\right)=(0,2)$. The tracer particles had a very large velocity for the helium injection. Therefore, they did not track the injection flow and penetrated the barrel shock wave

The measurement plane was located on the center line of the lower wall. Therefore, it could be assumed to be a plane of symmetry in the mean flowfield and the mean spanwise velocity component $\bar{w}$ would be zero. For both injection species, the measured mean spanwise velocity was smaller than the spanwise uncertainty.

In the air injection case, the mean streamwise velocity component $\bar{u}$ shown in Fig. 3 (a), the injectant flow is slower than the mainstream velocity $\bar{u}_{\text {main }}$. On the other hand, in the helium injection case, the helium injection flow is faster than the mainstream velocity $\bar{u}_{\text {main }}$. This difference between the mean velocity fields affected the turbulence properties as discussed in the next section.

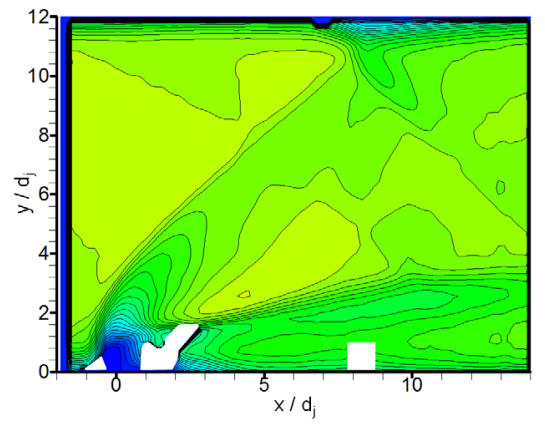

(a) Sreamwise component $\bar{u} / \bar{u}$ main

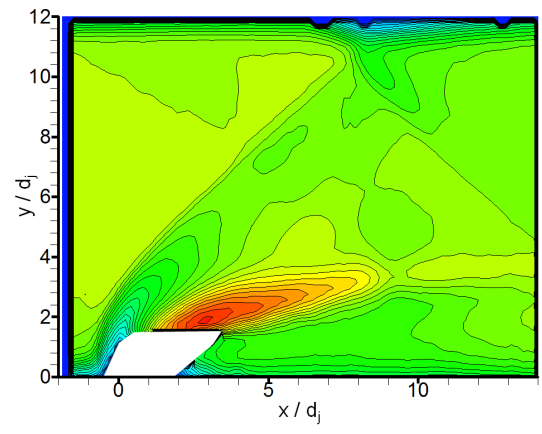

(a) Sreamwise component $\bar{u} / \bar{u}_{\text {main }}$

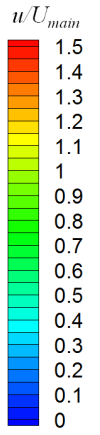

0 


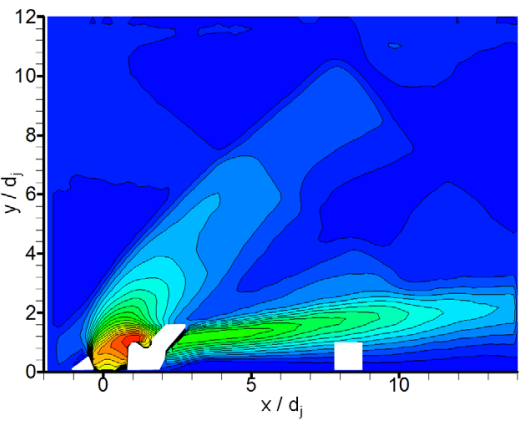

(b) Heightwise component $\bar{v} / \bar{u}$ main

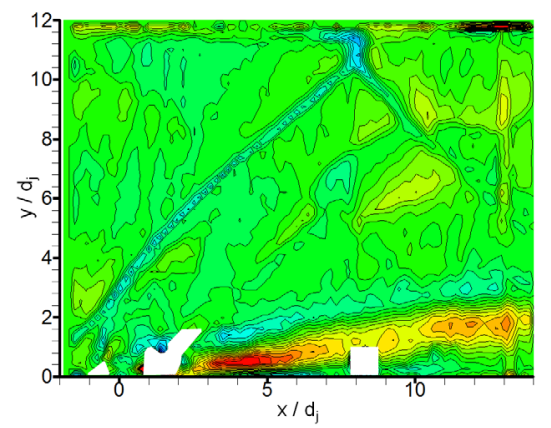

(c) Spanwise component $\bar{w} / \bar{u}_{\text {main }}$

Fig. 3 Mean velocity distribution for air injection

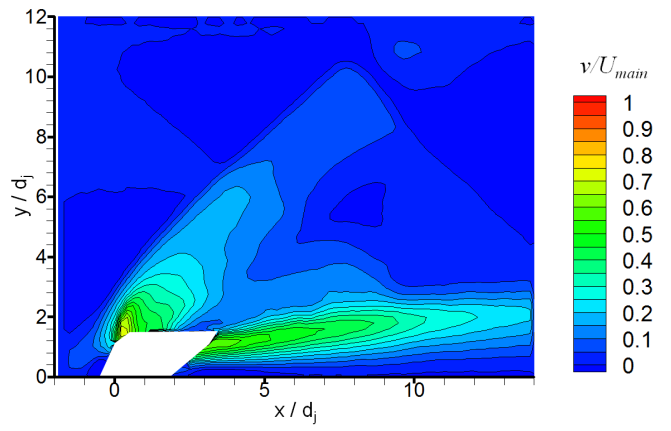

(b) Heightwise component $\bar{v} / \bar{u}$ main

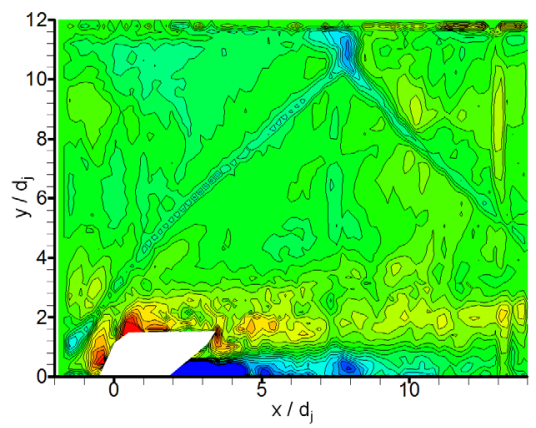

(c) Spanwise component $\bar{w} / \bar{u}$ main

Fig. 4 Mean velocity distribution for helium injection

\section{Velocity fluctuation distributions}

Figures 5 and 6 show the velocity fluctuation intensity distribution of each component. The intensities were nondimensionalized using the difference between the injection speed $V_{j e t}$ and the mainstream velocity $\bar{u}_{\text {main }}$. By using this reference velocity, some influences of the injection velocities might be removed.

The dashed-dotted and the two point dashed-dotted lines indicate, respectively, the $10 \%$ and 50\% averaged concentration tracks measured by Takahashi et al. [2]. The maximum averaged concentration track is not shown, because it is almost the same as the red line that indicates the maximum track of mean streamwise velocity gradient.

The 50\% averaged concentration track represented the most intensive concentration of jet fluctuations [2]. Therefore, this track represents the track of the maximum concentration gradient. However, in this study, this track did not coincide with the track of the maximum streamwise velocity gradient. Then, we considered that the expansion condition of the jet. It expanded non-uniformly before it passed through the Mach disk. In the upstream side, the jet did not expand so much because the pressure of air stream ahead of the jet was high due to passage of the bow shock wave. On the other hand, the jet expanded so much because the pressure of air stream back of the jet was low. Mach number of the jet was low in the upstream side and high in the downstream side. Then, this Mach number distribution resulted in high stagnation pressure in the upstream side and low one in the downstream side. Therefore, the jet velocity is fast in the mainflow side and is slow in the wall side. However, this phenomenon did not affect to the concentration field. Thus, the $50 \%$ averaged concentration track did not coincide with the track of the maximum streamwise velocity gradient. 


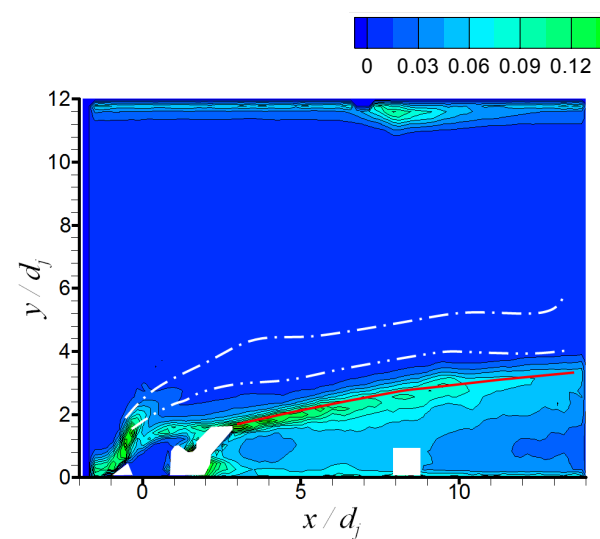

(a) $\overline{u^{\prime 2}} /\left(V_{\text {jet }}-\bar{u}_{\text {main }}\right)^{2}$

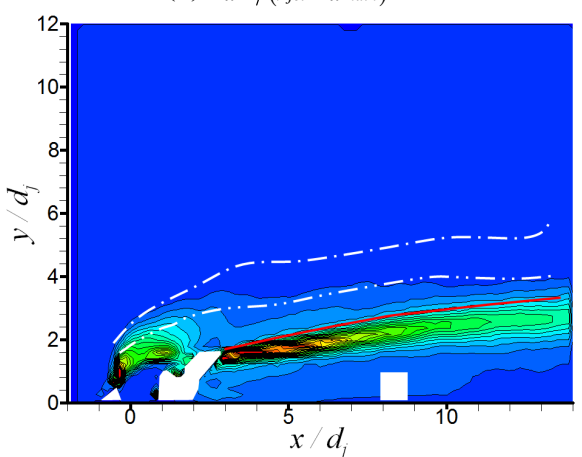

(b) $\overline{v^{\prime 2}} /\left(v_{\text {jet }}-\bar{u}_{\text {main }}\right)^{2}$

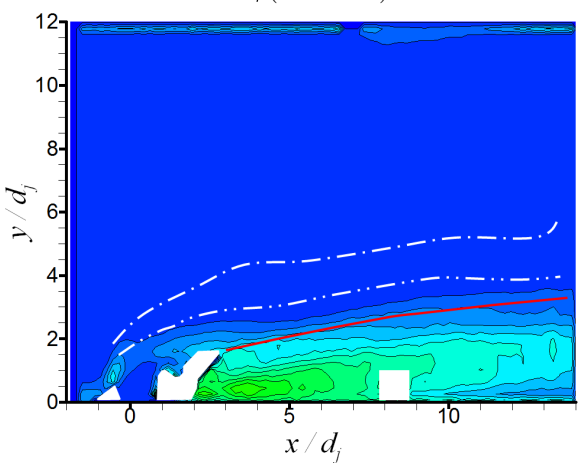

(c) $\overline{w^{\prime 2}} /\left(V_{\text {jet }}-\bar{u}_{\text {main }}\right)^{2}$

Fig. 5 Velocity fluctuation intensity distribution for air injection

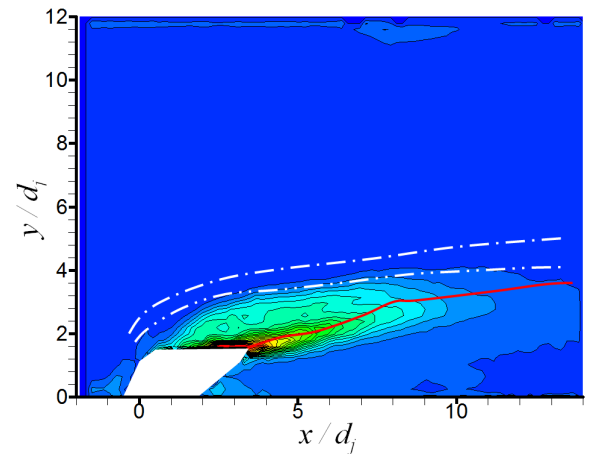

(a) $\overline{u^{\prime 2}} /\left(V_{\text {jet }}-\bar{u}_{\text {main }}\right)^{2}$

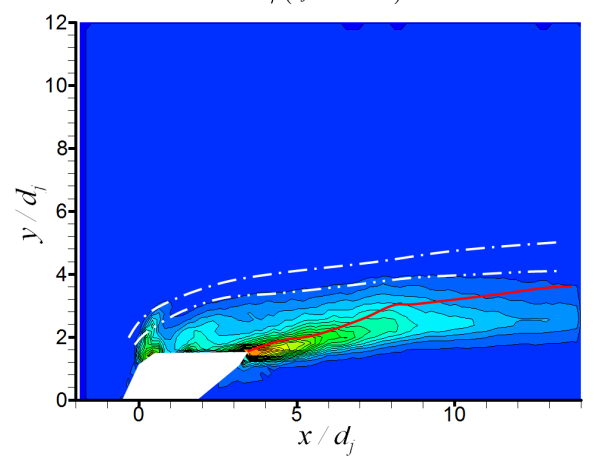

(b) $\overline{v^{\prime 2}} /\left(v_{\text {jet }}-\bar{u}_{\text {main }}\right)^{2}$

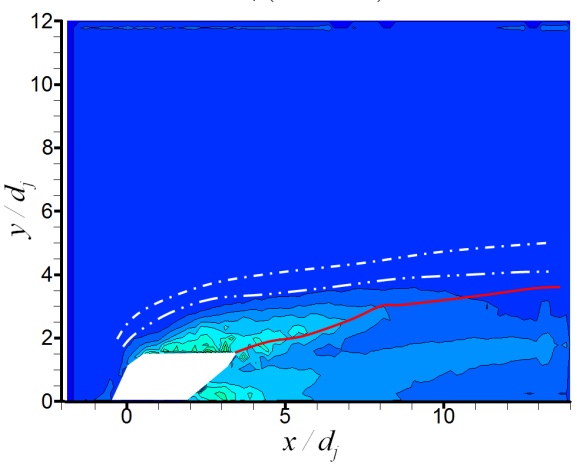

(c) $\overline{w^{\prime 2}} /\left(V_{\text {jet }}-\bar{u}_{\text {main }}\right)^{2}$

Fig. 6 Velocity fluctuation intensity distribution for helium injection

For the air injection case, the streamwise velocity fluctuation intensity, shown in Fig. 5 (a), spreads downstream and under the jet. For the helium injection case, however, the streamwise velocity fluctuation intensity, shown in Fig. 6 (a), does not spread downstream and under the jet. Instead the spread is around the mean streamwise velocity gradient. The heightwise velocity fluctuation intensity, shown in Figs. 5 and 6 (b), also spreads downstream more for air than for helium injection. The velocity and density differences between the mainstream and the jet were smaller for air injection than for helium injection. Therefore, in the air injection case, mass diffusion of injectant gas hard to mix with the main flow than the helium injection case.

For air injection, the velocity fluctuations were large behind the bow shock wave near the wall (Fig. 5) but for helium injection they were not so large (Fig. 6). The cause was the jet boundary fluctuation, the bow shock wave fluctuation or the barrel shock wave fluctuation. However, we could not determine which, because the resolution of this measurement was too rough to capture this phenomenon. 
For both injectant species, the spanwise velocity fluctuation intensity (Figs. 5 and 6 (c)) was large under the jet, because the jet and CVP vibrated along the $z$ direction.

The track of the maximum streamwise velocity gradient was nearly equal to the track of the maximum streamwise velocity fluctuation intensity. Furthermore, this track located higher than that of the maximum heightwise velocity fluctuation intensity. The value of spanwise velocity fluctuation intensity equals to streamwise velocity fluctuation intensity.

The track of the maximum spanwise velocity gradient existed near the wall. This phenomenon agreed qualitatively with subsonic injection flow [12].

Figures 7 and 8 show distributions of the Reynolds stress $-\overline{u^{\prime} v^{\prime}}$ for both injection cases. Since we did not measure instantaneous density in this study, we could not obtain the true Reynolds stress $-\overline{\rho u^{\prime} v^{\prime}}$. Instead, we use $-\overline{u^{\prime} v^{\prime}}$ as the Reynolds stress. In the air injection case, the Reynolds stress $-\overline{u^{\prime} v^{\prime}}$ was high behind the bow shock wave and along the track of the maximum mean streamwise velocity gradient (red line). The cause of the Reynolds stress $-\overline{u^{\prime} v^{\prime}}$ behind the bow shock wave was the same as that for the phenomenon occurring in the velocity fluctuation distribution behind the bow shock wave near the wall (see Fig. 5).

Along the track of the maximum mean streamwise velocity gradient (solid red line), a shear stress was produced by the mean shear between the injectant flow and mainflow. So a positive Reynolds stress occurs along this line. In the helium injection case, the injectant flow was faster than the mainflow. So, negative Reynolds stress regions appeared along the track of the maximum mean velocity gradient (shown as a dashed red line in Fig. 8).

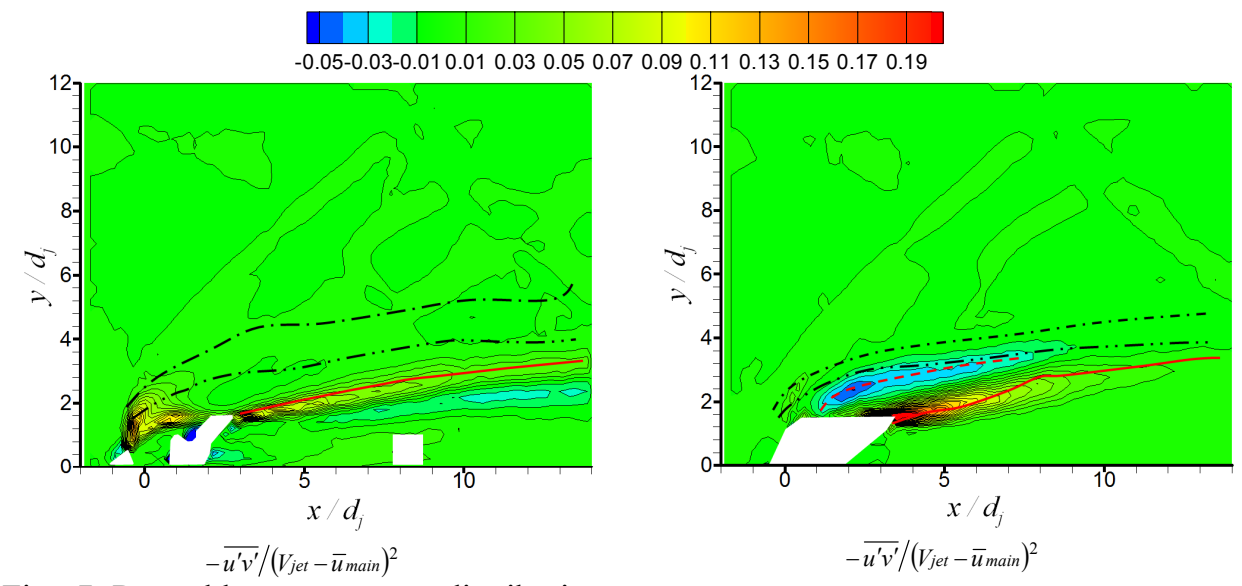

Fig. 7 Reynolds stress $-\overline{u^{\prime} v^{\prime}}$ distribution for air injection

Fig. 8 Reynolds stress $-\overline{u^{\prime} v^{\prime}}$ distribution for helium injection

\section{Single-time two-point spatial correlation}

The single-time two-point spatial correlation $C u_{i} u_{j}$ is given by

$$
\begin{aligned}
& C_{u_{i} u_{j}}(\Delta x, \Delta y)=\frac{\frac{1}{N} \sum_{n=1}^{N}\left[u_{i, n}^{\prime}(x, y) \cdot u_{j, n}^{\prime}(x+\Delta x, y+\Delta y)\right]}{u_{i_{-} r m s}(x, y) \cdot u_{j_{-} r m s}(x+\Delta x, y+\Delta y)} \\
& u_{i}^{\prime} \quad: \text { instantaneous velocity fluctuation of } u_{i} \\
& u_{i_{-} r m s}^{\prime}: \text { root-mean-square of } u_{i}^{\prime}
\end{aligned}
$$

In Eq. (1), point $(x, y)$ is the location of the reference point and $\Delta x$ and $\Delta y$ are the streamwise and transverse distances from the reference point, respectively.

In this study, the number of picture pairs in the sample was about 1500. For this sample size, a high correlation was where the absolute value of the correlation was $\left|C u_{i} u_{j}\right| \geq 0.1$, as calculated by a significance test [10].

We took the reference points on the $50 \%$ averaged concentration track that are reported 
by Takahashi et al. [2] because the large structure of concentration was produced by the velocity difference between the mainflow and the jet. Furthermore, we compared the spatial correlation of concentration and velocity fluctuation. In this paper, we discuss the reference point $\left(x / d_{j}, y / d_{j}\right)=(7,3.5)$, because the spatial correlations of velocity fluctuations at this point had characteristic structures of this flow field.

Figures 9 and 10 show the spatial correlation distributions for the two gas injection cases. The dotted line and dashed line indicate the bow shock wave and the reflected shock wave, respectively. The dashed-dotted and the two point dashed-dotted lines indicate the $10 \%$ and $50 \%$ averaged concentration tracks measured by Takahashi et al. [2], respectively. The red solid line indicates the track of the maximum mean streamwise velocity gradient.

In the air injection case, $C_{u u}$ had a narrow positive correlation region (Fig. 9 (a)), but in the helium injection case, $C_{u u}$ had a positive correlation region elongated along the $50 \%$ averaged concentration track (two point dashed-dotted line in Fig. 10 (a)). This difference in the $C_{u u}$ correlation regions for the air and helium injection cases is similar to ones found in the scalar correlation regions [2]. Along the 50\% averaged concentration track, the injectant flow and the mainflow alternate. Figures 11 and 12 are maps of the instantaneous streamwise velocity fluctuations and they show alternate positive and negative velocity fluctuation regions along the 50\% averaged concentration track. The injectant flow formed a bump-like structure, and the velocity fluctuation was almost constant in this region. The structure is longer and bigger in the helium injection case than in the air injection case. Therefore, the helium $C_{u u}$ region is longer than the one for air. $C_{u u}$ is negative in a region around $\left(x / d_{j}, y / d_{j}\right)=(6,4.5)$ in the helium injection case (Fig. 10 (a)). The velocity of the helium jet was considerably faster than the mainflow. So, when the bow shock wave moved forward due to deeper penetration of the jet, the velocity near the bow shock wave was decreased. At the same time, the injectant flow sped up, and the instantaneous velocity in the region around $\left(x / d_{j}, y / d_{j}\right)=(6,4.5)$ was higher than the mean velocity. Thus, a negative correlation region was formed.

Both injectant cases showed high correlation regions of heightwise velocity fluctuation around the reference point at $x / d_{j}=7$ on the $50 \%$ averaged concentration track (see Fig. 9 (b) and Fig. 10 (b)). The shapes of the correlation regions were vertically elongated ellipses. Around a point $\left(x / d_{j}, y / d_{j}\right)=(5,6)$, positively and negatively correlated regions existed for both injection cases. We think that these regions represent a sound wave irradiated by the motion of the jet plume. When a bump-like structure appeared in the jet plume above the injection port with a positive fluctuation of the vertical velocity component, its influence propagated in the mainflow as a pressure wave at a sonic speed relative to the fluid motion. Propagation of the wave was bounded by the bow shock wave. The bump-like structure flowed downstream at the velocity of the jet plume. For example, Fig. 13 shows a schlieren image of a similar transverse injection flowfield. In this figure, we can see that the sound wave propagates downstream from the outer boundary of the jet plume. In the air injection case, the injectant flow downstream of the Mach disc was slower than the mainflow, as shown in Fig. 3. Thus, the correlation region representing the sound wave was separated from the one around the reference point. On the other hand, in the helium injection case, the injectant flow was faster than the mainflow, as shown in Fig. 4, and the bump-like structure in the jet plume caught up with this wave, so the correlation region of the sonic wave was not separated from the one around the reference point. For these reasons, positive and negative correlation regions were formed. Since the injection velocity of helium was faster than that of air, the correlated region for helium injection was larger than that for air injection.

For air injection, the reattachment shock wave emanating near the reference point was observed and it was weakly correlated with the reference point (Fig. 9 (b)). On the other hand, for helium injection, the reattachment shock wave was not observed (Fig. 10 (b)). 
In Fig. 9 (c) and Fig. 10 (c), $C_{w w}$ was positive in a region along the 50\% averaged concentration track and negative under it. This indicates counter-rotating vortex pair (CVP). As previously mentioned, the jet vibrated in the $z$ direction and the CVP also vibrated in the $\mathrm{z}$ direction. The CVP had opposite velocities above and below, forming these correlation regions.

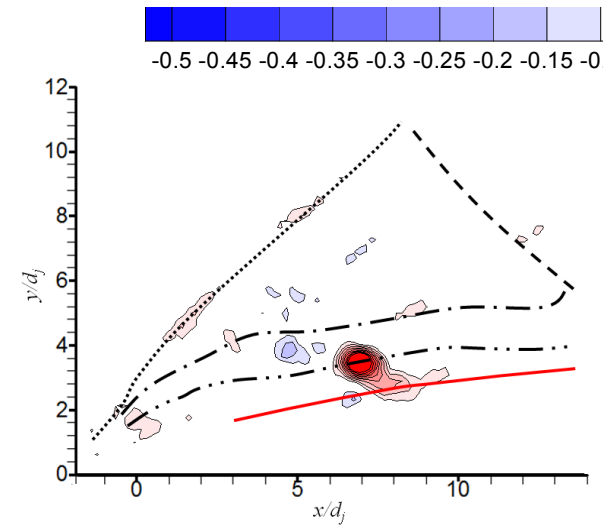

(a) $C_{u u}$

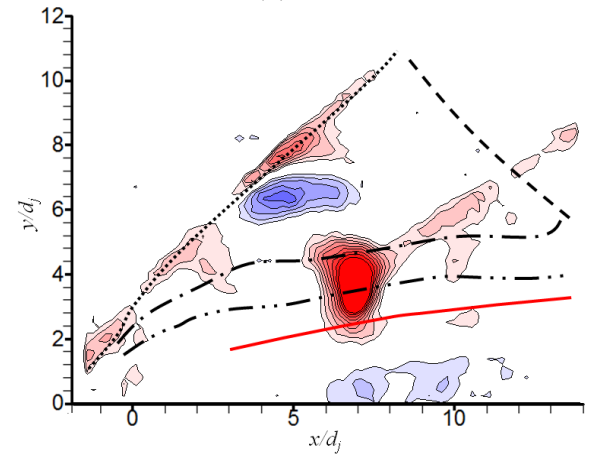

(b) $C_{v v}$

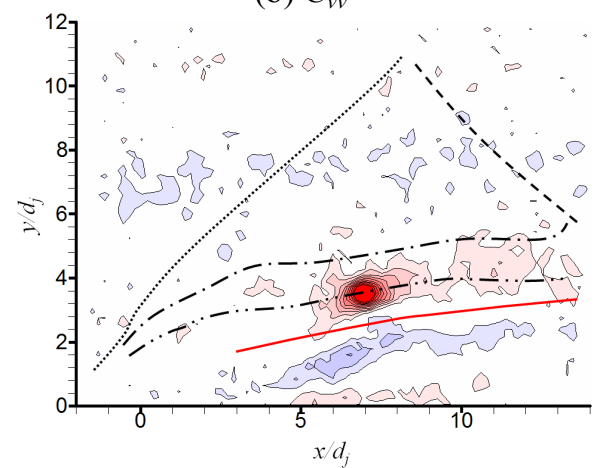

(c) $C_{w w}$

Fig. 9 Spatial correlation distribution for air injection.

Reference point: $\left(x / d_{j}, y / d_{j}\right)=(7,3.5)$.

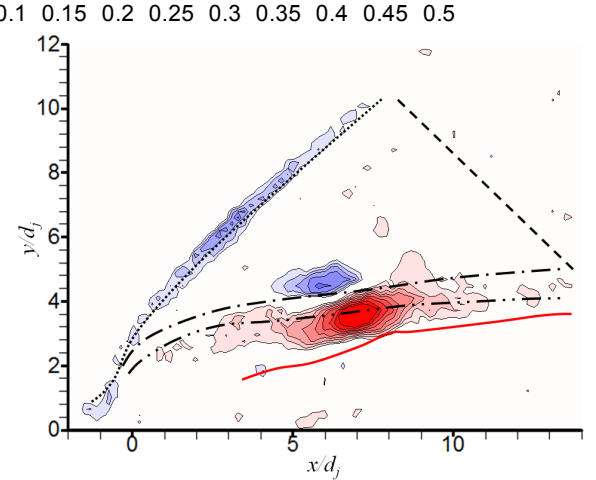

(a) $C_{u u}$

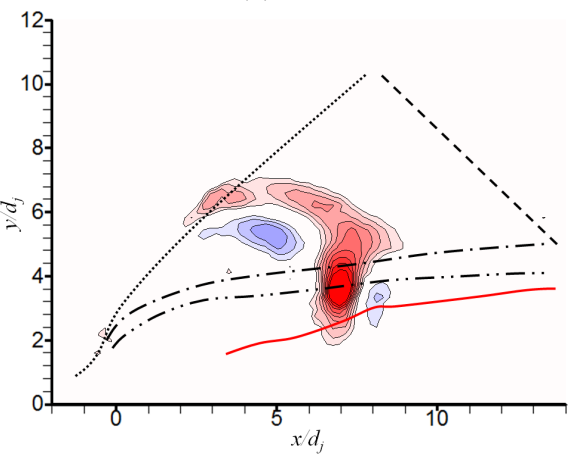

(b) $C_{v v}$

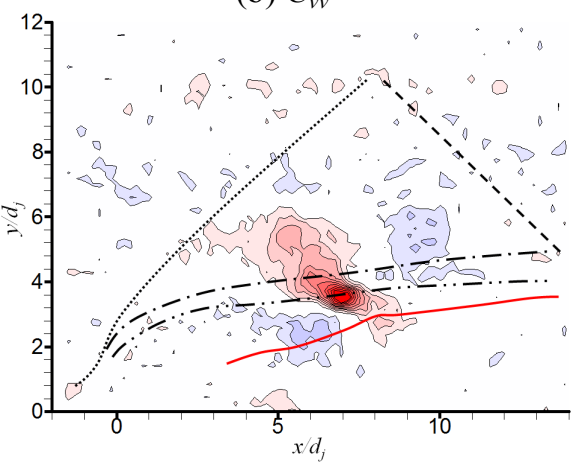

(c) $C_{w w}$

Fig. 10 Spatial correlation distribution for helium injection.

Reference point: $\left(x / d_{j}, y / d_{j}\right)=(7,3.5)$. 


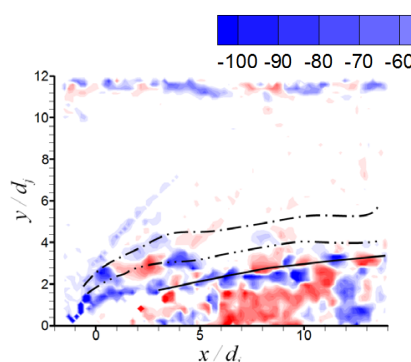

(a)

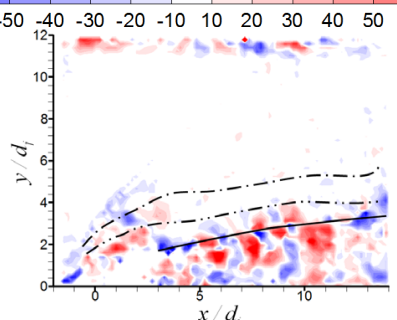

(b)

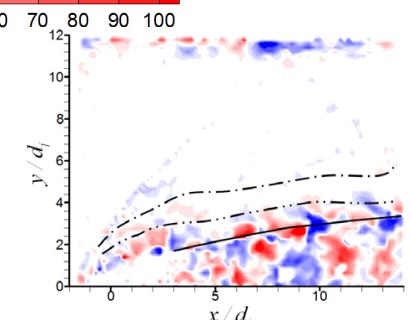

(c)

Fig. 11 Instantaneous velocity fluctuations $u^{\prime}$ for air injection (randomly selected)

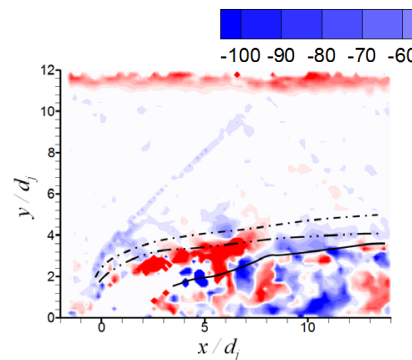

(a)

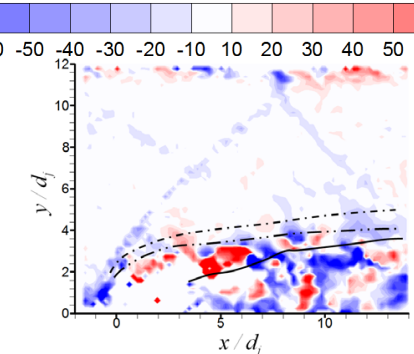

(b)

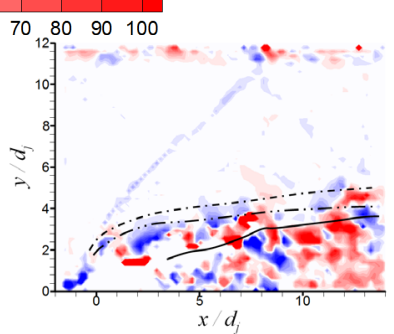

(c)

Fig. 12 Instantaneous velocity fluctuations $u^{\prime}$ for helium injection (randomly selected)

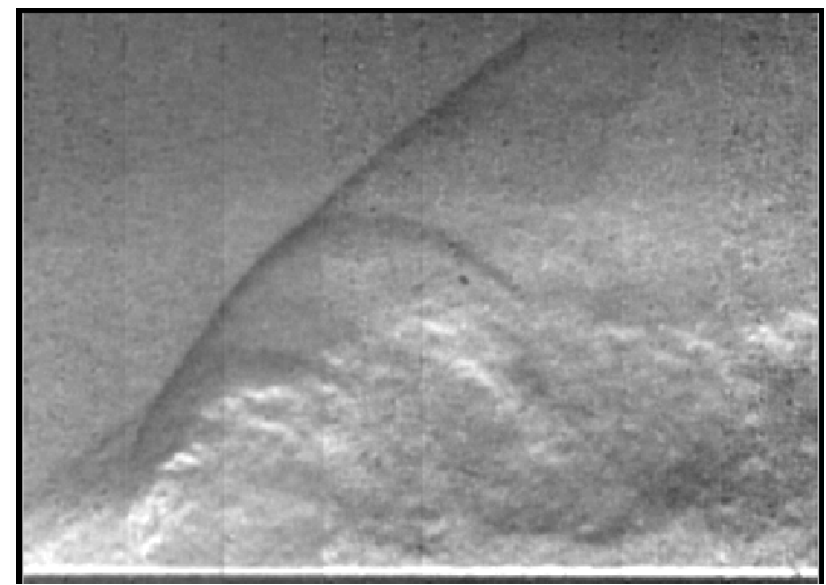

Fig. 13 Schlieren image of the transverse injection flow

\section{Concluding remarks}

We measured the supersonic flowfield with transverse injection by SPIV and calculated the single-time two-point spatial correlations of the velocity fluctuations. The following results were obtained.

$>$ We captured the bow shock wave formed by the injection and its reflection from the upper wall.

D CVP vibrated along the spanwise direction for both injection cases.

$>$ Reynolds stress was formed along the track of the maximum mean velocity gradient.

$>$ The $50 \%$ averaged concentration track measured by PLIF did not coincide with the track of the maximum streamwise velocity gradient.

$>$ A strong correlation region existed around the reference point and its shape changed with the selected velocity components in both injection cases.

$>$ For helium injection, the single-time two-point spatial correlation showed a large scale motion along the $50 \%$ averaged track. 
A sound wave irradiated by the motion of the jet plume was observed between the reference point and the bow shock wave.

\section{Acknowledgment}

This work was supported by the MEXT in Japan, Grant-in-Aid for Scientific Research (B), 20360081 and 23360377.

\section{References}

[1] Gruber, M. R., Nejad, A. S.; "Compressibility effects in supersonic transverse injection flowfields," Physics of Fluids, Vol. 9, No. 5, 1997, pp. 1448-1461.

[2] Takahashi, H., Oso, H., Kouchi, T., Masuya, G.; "Scalar Spatial Correlations in a Supersonic Mixing Flowfield," AIAA Journal, Vol. 48, No. 2, 2010, pp. 443-452.

[3] Takahashi, H., Masuya, G., Hirota, M.; "Effects of Injection and Main Flow Conditions on Supersonic Turbulent Mixing Structure," AIAA Journal, Vol. 48, No. 8, 2010, pp. 1748-1756.

[4] Melling, A., "Tracer Particles and Seeding for Particle Image Velocimetry," Measurement Science and Technology, Vol. 8, No. 12, 1997, pp. 1406-1416.

[5] Kähler, C. J., Sammler, B., Kompenhans, J., "Generation and Control of Tracer Particles for Optical Flow Investigations in Air," Experiments in Fluids, Vol. 33, 2002, pp. 736-742.

[6] Kitamura, E., Matsumoto, M., Koike, S., Masuya, G., "PIV Measurement of Supersonic Swirling Jet," Proceedings of the 30th Symposium on Visualization, Visualization Society of Japan, Tokyo, 2002, pp. 189-192 (in Japanese).

[7] Koike, S., Takahashi, H., Tanaka, K., Hirota, M., Takita, K., Masuya, G., "Correction Method for Particle Velocimetry Data Based on the Stokes Drag Law," AIAA Journal, Vol. 45, No. 11, 2007, pp. 2770-2777.

[8] The visualization Society of Japan, PIV handbook in Japanese, Morikita, Tokyo, 2002, pp. 138-164, pp. 166-169.

[9] Hinze, J. O., Turbulence, McGraw-Hill, New York, 1959, pp. 223-225

[10] Uramoto, S., Kouchi, T., Masuya, G.; "Stereoscopic PIV measurement of supersonic boundary layer," 42nd Fluid Dynamics Conference / Aerospace Numerical Simulation Symposium 2010, 2C3, June 2010 (in Japanese)

[11] http://www.tamagaki.com/math/Statistics605.html

[12] Yuan, L. L., Street, R. L. and Ferziger, J. H., "Large-eddy simulations of a round jet in crossflow," J. Fluid Mech., Vol. 379, 1999, pp. 71-104 